\title{
PROTOTIPO DE VEHÍCULO DE GUIADO AUTÓNOMO PARA SISTEMA DE ALMACENAMIENTO Y RECUPERACIÓN AUTOMÁTICO DE LIBROS
}

\author{
PROTOTYPE OF AUTONOMOUS GUIDED VEHICLE FOR AUTOMATIC \\ BOOKS STORAGE AND RETRIEVAL
}

Gustavo Meneses Benavides ${ }^{1}$, Camilo Velasquez Gómez ${ }^{2}$, Richard Grisales Arboleda ${ }^{3}$

${ }^{1}$ Ingeniería Electrónica,Universidad de San Buenaventura Medellín, Medellín, Colombia, gmeneses23@gmail.com

2. Ingeniería Electrónica Universidad de San Buenaventura Medellín, Medellín, Colombia, velasquezgomez8@hotmail.com

3. Ingeniería Electrónica Universidad de San Buenaventura Medellín, Medellín, Colombia, r.grisales07@gmail.com

Recibido: 10 de febrero de 2014

Aceptado: 15 de marzo de 2014

*Correspondencia del autor. Gustavo Meneses Benavides, Universidad de San Buenaventura Medellín,Medellín, Colombia

Email: gmeneses23@gmail.com

\begin{abstract}
RESUMEN
Se presenta el proceso de diseño e implementación de un prototipo de vehículo de guiado autónomo (AGV por sus siglas en inglés) que debe realizar tareas de ubicación/recolección automática de libros en un ambiente de pruebas bajo condiciones controladas. El prototipo de AGV actúa como el elemento central de un sistema de almacenamiento y recuperación automático en el que también se integra la tecnología de identificación por radiofrecuencia con elementos mecatrónicos, de sensórica y de comunicaciones inalámbricas, mediados por interfaces de usuario para el monitoreo del vehículo y el control de inventario. El protocolo de comunicación MiWi P2P, que está basado en el estándar IEEE 802.15.4, se ha elegido para cubrir las comunicaciones inalámbricas del sistema en el ambiente de prueba.
\end{abstract}

\section{Palabras clave}

Identificación por Radiofrecuencia (RFID), Sistema de almacenamiento y recuperación automático (ASRS), Vehículo de Guiado Autónomo (AGV), protocolo MiWi P2P.

\begin{abstract}
:
The objective of this article is to present the process of designing and implementing a prototype of autonomous guided vehicle (AGV) that must carry out automatic tasks of gathering and location of books in a test environments under controlled conditions. The AGV prototype is a central element of an automatic storage and retrieval system and involves the radiofrequency identification technology with mechatronic elements, of sensors and wireless communication mediated by user interfaces for vehicle monitoring and inventory control. The MiWi P2P communication protocol, which is based on the IEEE 802.15.4 standard, has been chosen to cover wireless communications system in the test environment.
\end{abstract}

\section{Key words:}

Radio Frequency Identification (RFID), and automatic storage system recovery (ASRS), Autonomous Guided Vehicle (AGV), MiWi P2P protocol. 


\section{INTRODUCCIÓN}

Durante las últimas décadas los sistemas automatizados se han integrado a tareas de almacenamiento/ recuperación de objetos a nivel industrial en aplicaciones como las de manejo logístico de mercancía y de distribución de productos [1], adicionalmente este tipo de sistemas también se pueden encontrar en aplicaciones fuera del campo industrial, como en bibliotecas, siendo integrados al manejo de los libros que circulan hacia el interior o el exterior de estas.

Los ASRS (sistemas de almacenamiento y recuperación automática por sus siglas en inglés) pueden tener dimensiones muy grandes dependiendo del tipo de aplicación y de factores como la cantidad de inventario que debe ser movilizado, no obstante puede haber sistemas de dimensiones menores aplicados en entornos con necesidades menos exigentes que las del campo industrial o de las grandes bibliotecas mundiales [2].

Los vehículos de guiado autónomo son un tipo especial de dispositivo robótico móvil que realiza actividades relacionadas con la recuperación, transporte y ubicación de objetos sin la intervención directa de personas [3]. Para lograr su cometido los AGV se apoyan en sensores y otros medios electrónicos para guiarse por rutas pre-establecidas o que pueden ser programadas dependiendo de las tareas que deben realizarse y los recorridos que deben cubrirse. E1 AGV puede recuperar y ubicar objetos de inventario utilizando dispositivos mecatrónicos acoplados a su estructura, es así como pueden actuar como robots en el entorno de un ASRS.

\section{DISEÑO DEL PROTOTIPO DE VEHÍCULO DE GUIADO AUTÓNOMO, AGV}

\section{A. Definición del tipo constructivo para el prototi- po de AGV}

Luego de establecer los requerimientos del sistema mecatrónico a desarrollar, se consideraron diferentes opciones a ser adoptadas para la estructura que define el prototipo AGV. Los diferentes borradores de diseño fueron evaluados por las personas asociadas al proyecto, teniendo en cuenta elementos técnicos como el diseño del mecanismo de guiado, la escogencia del tipo de baterías y de motores, los requerimientos de movimiento y las restricciones de tamaño, peso y con- sumo de energía. Igualmente los hallazgos arrojados por algunas pruebas realizadas preliminarmente ayudaron a definir las características del tipo de diseño elegido, Fig. 1.

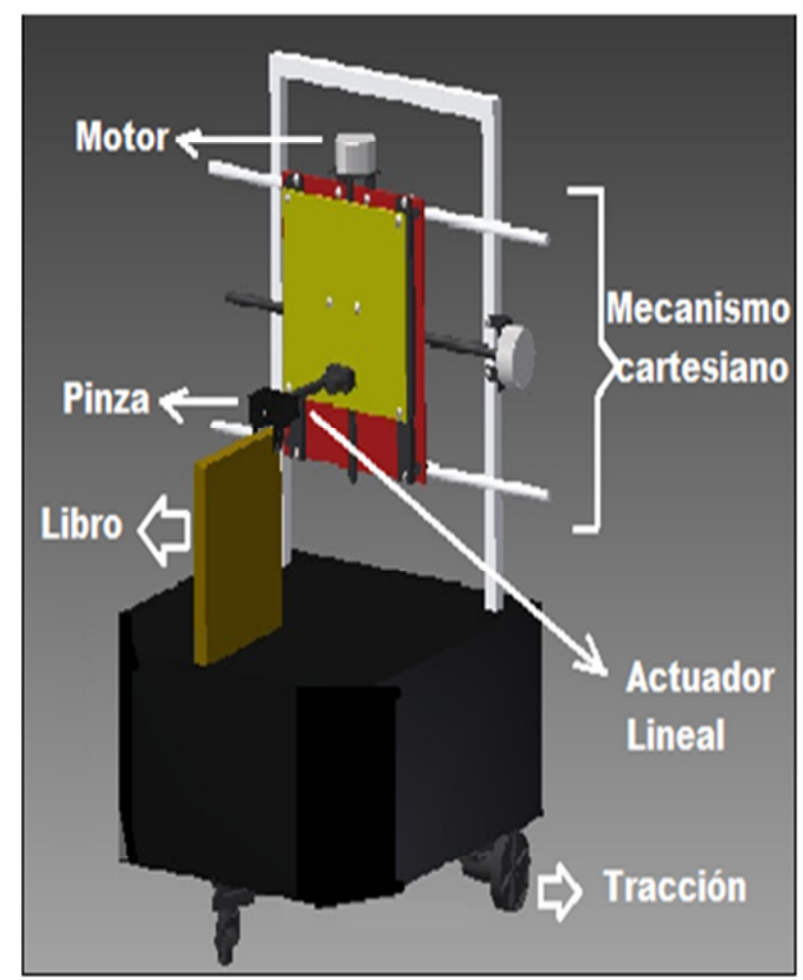

Fig. 1. Tipo constructivo elegido para el prototipo de AGV

Las dimensiones del prototipo AGV están definidas en concordancia con los pesos a transportar y los torques a desarrollar durante el proceso de almacenamiento/recuperación.

\section{B. Estructura del efector del prototipo AGV y otros aspectos complementarios}

Después de analizar diferentes configuraciones para el efector del AGV, se optó por implementar un vehículo que se apoya en un ensamble de actuador lineal con pinza que está acoplado a un mecanismo cartesiano para cumplir con los movimientos que se necesitan para hacer las funciones de almacenamiento/recuperación asignadas. Esta configuración nos permite obtener 3 grados de libertad; eje X, eje Y y profundidad (eje Z). El prototipo de vehículo de guiado autónomo utiliza para su desplazamiento un mecanismo con tracción en las ruedas traseras y una rueda libre en la parte delantera, todo el sistema es gobernado por tarjetas electrónicas que gestionan los sensores, los motores y las comunicaciones. 


\section{SISTEMAS DE COMUNICACIONES}

\section{A. Transmisión y recepción de la información del inventario.}

Para la identificación de los libros se utiliza la tecnología de RFID con etiquetas pasivas de lecto-escritura $(\mathrm{R} / \mathrm{W})$, de costo moderado y que se acceden bajo las especificaciones de los sistemas ISO15693 [4]. Se muestra, Fig. 2, el formato de comunicación para programar los tags con la información de los libros y para su lectura, durante las tareas de almacenamiento/recolección. La comunicación se realiza de manera serial, entre un microcontrolador y el módulo R/W RFID, que se adapta a la pinza del efector del AGV.
Luego de ser adquirida, la información de las etiquetas es empaquetada y transmitida hacia un punto ("nodo") de recepción de datos dentro del ASRS, para ello se utiliza el protocolo MiWi P2P. Microcontroladores de 8 bits como el PIC18F4620 de Microchip permiten implementar MiWi P2P, un protocolo basado en el estándar de comunicación para redes inalámbricas de área personal IEEE 802.15.4 [5]. Se muestra, Fig. 3, la red conformada por tres nodos que se comunican utilizando una topología P2P, en esta el nodo que transfiere los datos al computador es el coordinador de red (dispositivo de funciones completas, FFD) y los otros dos nodos son dispositivos de funciones reducidas (RFD) como establece el estándar IEEE 802.15.4 y, a su vez, MiWi P2P.

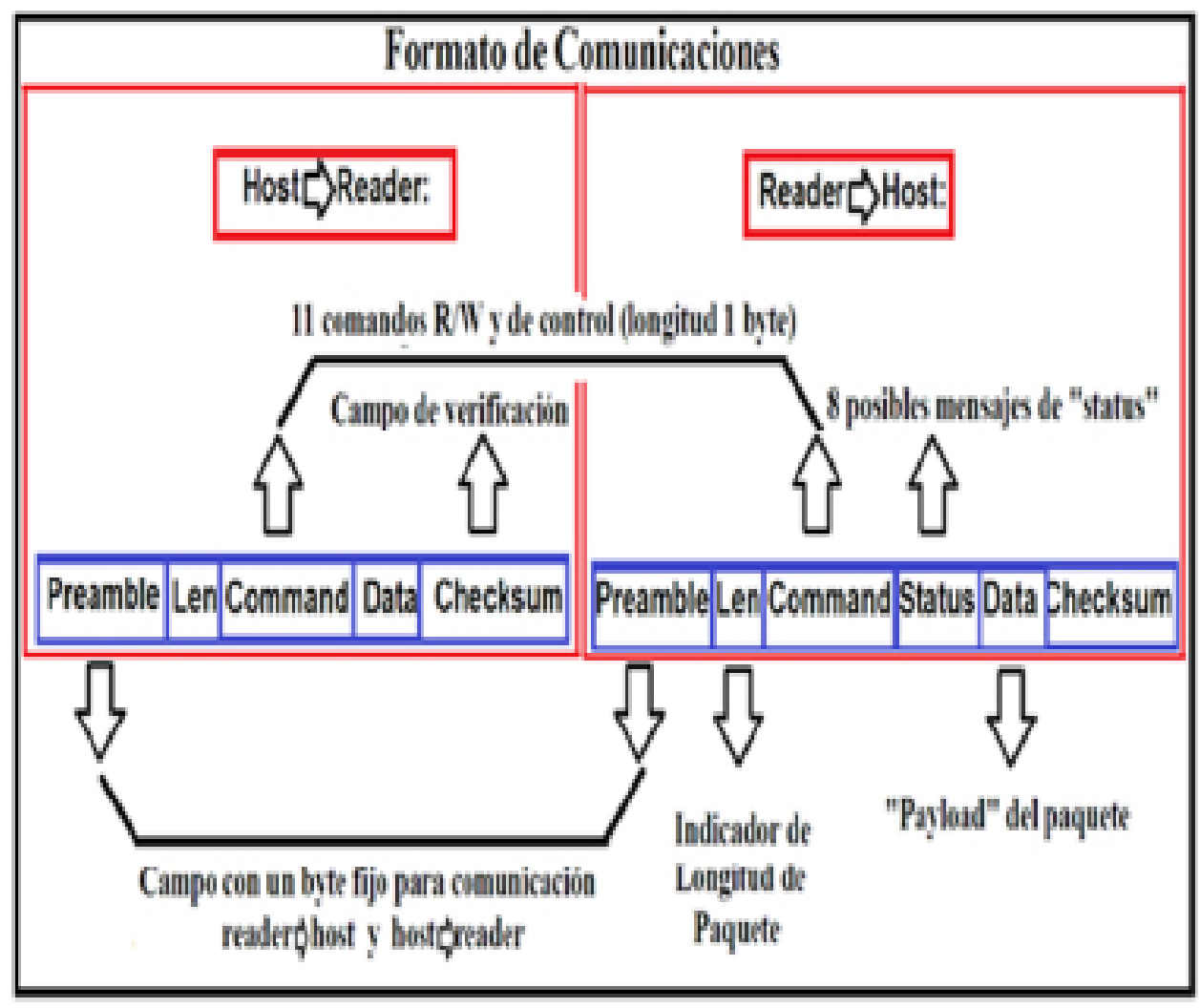

Fig. 2. Formato utilizado por el módulo RFID para la lectura/escritura de las etiquetas que identifican los libros 


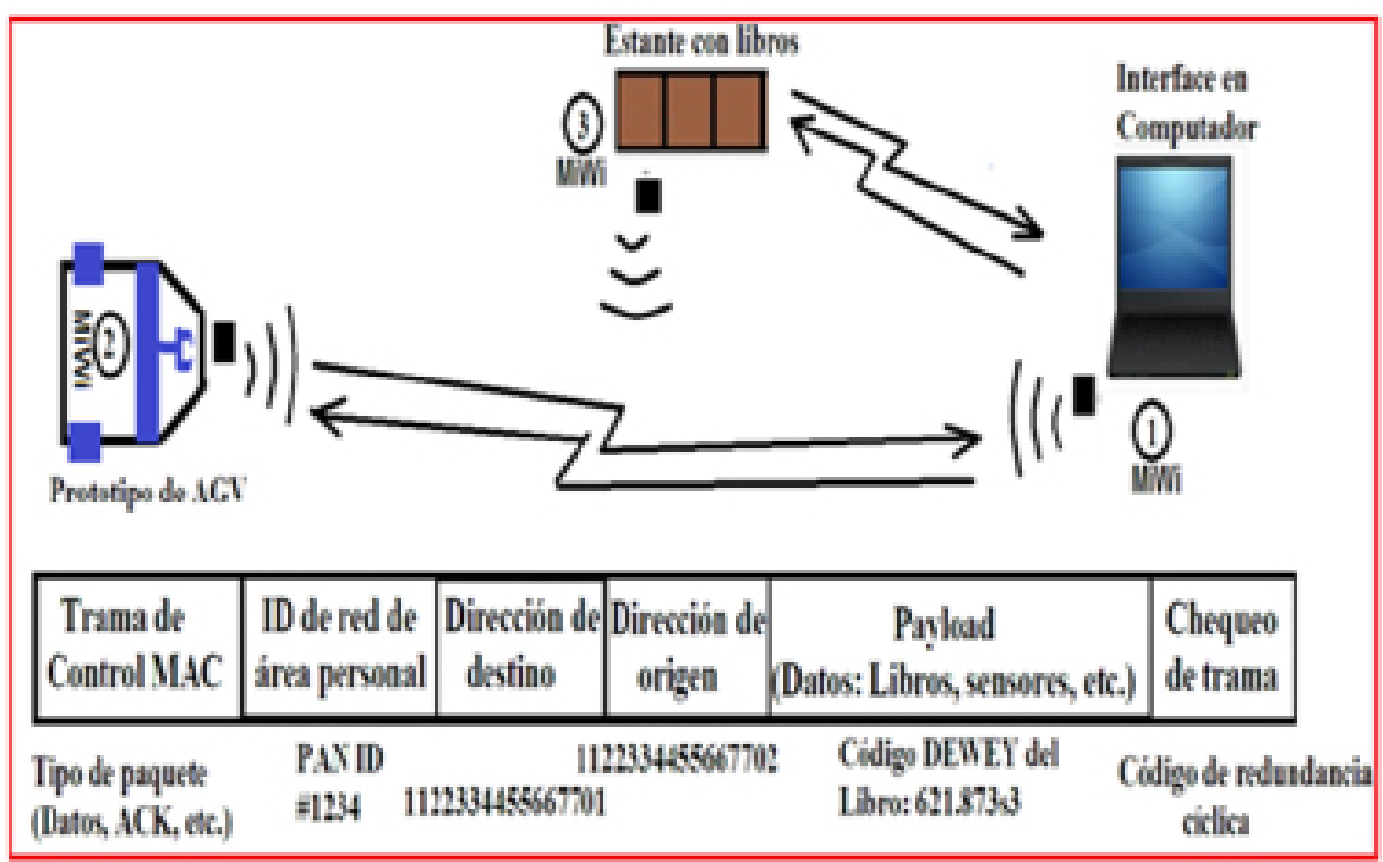

Fig. 3. Esquema de transmisión de paquetes para los nodos del ASRS utilizando el protocolo MiWi P2P

MiWi P2P permite utilizar la generalidad de las cualidades del estándar IEEE 802.15.4, con una robustez suficiente para aplicaciones que permiten escalabilidad e integración rápida de nuevos nodos, sin el pago de royalties.

\section{B. Comunicaciones por medios guiados y otras co- municaciones complementarias}

Para cubrir los requerimientos técnicos de operación del prototipo de AGV debemos apoyarnos en comunicaciones por medio guiado, para sus dispositivos asociados como sensores, transceptores y periféricos (UART, SPI e I2C), Fig. 4. De manera complementaria en el sistema ASRS se integra la transmisión inalámbrica de video en la banda de $2.4 \mathrm{GHz}$, este es captado desde el efector del AGV con el fin de ejecutar un algoritmo de procesamiento de imágenes que permita ubicar y recuperar con precisión los libros. Adicionalmente se plantea la integración de módulos serial/Bluetooth, que sirven como gateways para la transmisión de datos mediante el protocolo IEEE 802.15.1. El objetivo de los gateways, implementados con módulos transceptores RN41 o RN42 [6], es proveer acceso a los datos del AGV y del ASRS desde dispositivos móviles.

\section{INTERFACES Y ADMINISTRACIÓN DE LOS DATOS}

El sistema ASRS arroja un conjunto considerable de datos durante su operación, algunos datos se podrían denominar "técnicos", en su mayoría entregados por el vehículo de guiado autónomo, $\mathrm{y}$ otros que sirven de base para la consolidación de la información final presentada a los usuarios.

\section{A. Datos al interior del ASRS}

El conjunto de los datos arrojados por los sensores no constituyen en sí, por si solos, información de utilidad para el sistema. Estos datos deben procesarse y tratarse según su naturaleza para realizar tareas de control, navegación y referenciación coordenada del prototipo de $\mathrm{AGV}$, entre otros.

Las interfaces de usuario que se ejecutan en computadores u otros dispositivos con altas capacidades de procesamiento permiten gestionar el volumen de datos producido, dándole a este el uso y el destino adecuado. Posterior a la etapa de procesamiento, los datos se constituyen en información relativa al inventario, a la ubicación en un mapa de la posición actual del AGV y al registro de las tareas realizadas y de los eventos ocurridos en el sistema. 


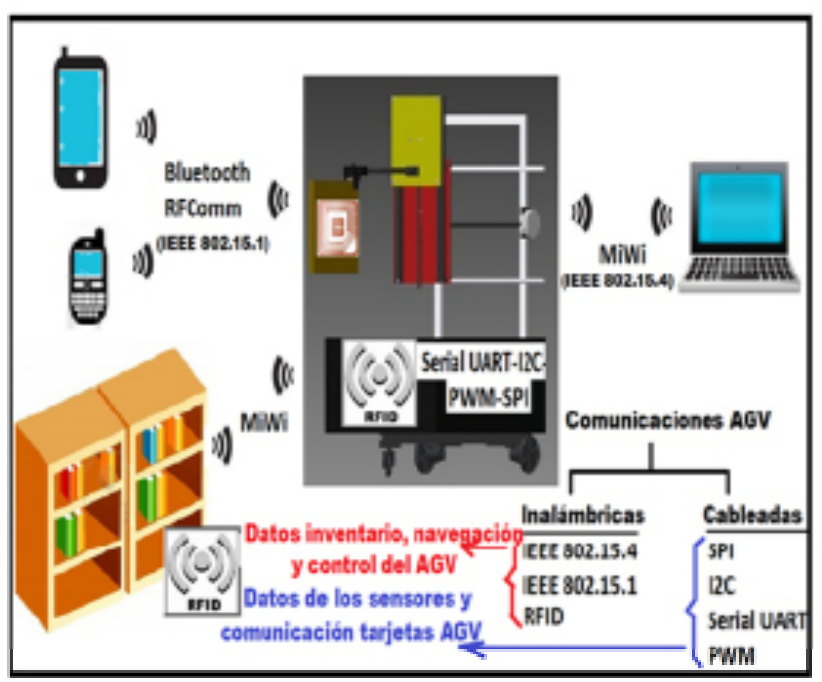

Fig. 4. Tráfico de datos técnicos al interior del ASRS y medio por el cual se transmiten

\section{B. Interfaces de usuario para un ASRS integrado}

Las interfaces y herramientas computacionales para el procesamiento y visualización de la información permiten, en complemento con el prototipo AGV, tener un sistema automático de ubicación/recuperación de libros que operará bajo condiciones controladas, es decir, en un escenario de prueba sin la presencia de personas ni interacción con personal no calificado. Se muestra el escenario general de operación del AGV y su integración con las interfaces de usuario y los demás elementos que permiten constituir un ASRS para libros, Fig. 5.



Fig. 5. Esquema general del sistema de almacenamiento y recuperación automático de libros

\section{RESULTADOS}

\section{A. Prototipo de vehículo de guiado autónomo}

En la fase de avance actual del proyecto se ha logrado construir un primer prototipo de vehículo guiado autónomo para desempeñarse en un ASRS para libros, Fig. 6. A partir de la estructura mecátronica desarrollada se puede proceder a la integración de los sistemas electrónicos de navegación y control del AGV para realizar pruebas de operación del prototipo en el ambiente con condiciones controladas que se ha establecido para fines de experimentación.

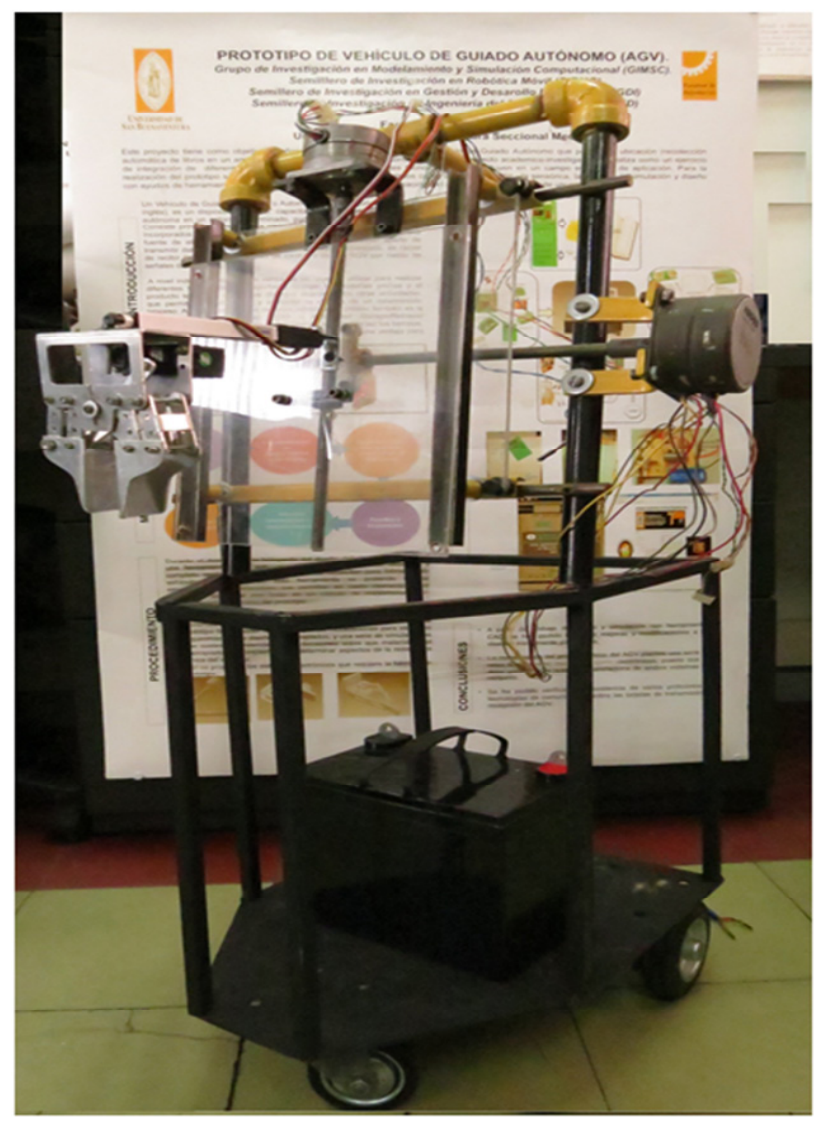

Fig. 6. Prototipo experimental de vehículo guiado autónomo para ASRS de libros

\section{B. Tarjetas electrónicas}

Se han realizado tarjetas electrónicas sobre las cuales se han efectuado las respectivas pruebas para corregir algunos defectos de operación identificados, Fig. 7.

También se ha desarrollado el firmware que gobierna los microcontroladores alojados en las tarjetas, este firmware también está continuamente sujeto a tareas de depuración basadas en las pruebas realizadas y los resultados obtenidos. 

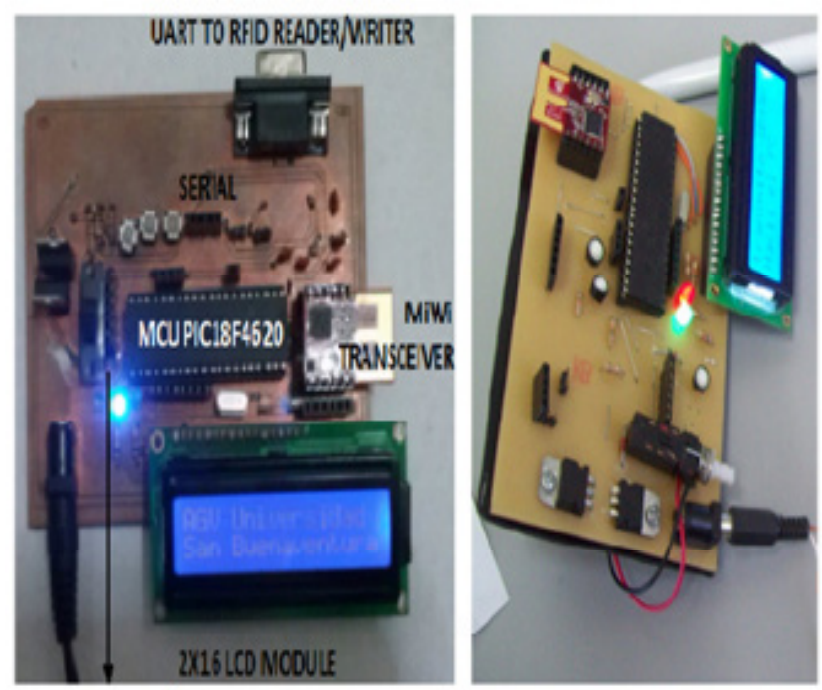

MCU DEBUGGING/PROGRAMMING PINS

Fig. 7. Tarjetas para control del AGV y comunicación con el computador central del ASRS

El prototipo de vehículo de guiado autónomo se apoya en un conjunto de sensores de diferente naturaleza para su funcionamiento. Una brújula digital, encoders acoplados a las ruedas de tracción del AGV y un arreglo de sensores de distancia por ultrasonido, desplegados en el ambiente de pruebas, se utilizan para levantar la información de las coordenadas de ubicación del AGV. Un sistema de seguidor de línea por infrarrojo se utiliza para seguir trayectorias predefinidas trazadas en el piso. Para el prototipo desarrollado se ha elegido el método de detección por ultrasonido para evitar el choque con obstáculos o con personas presentes.

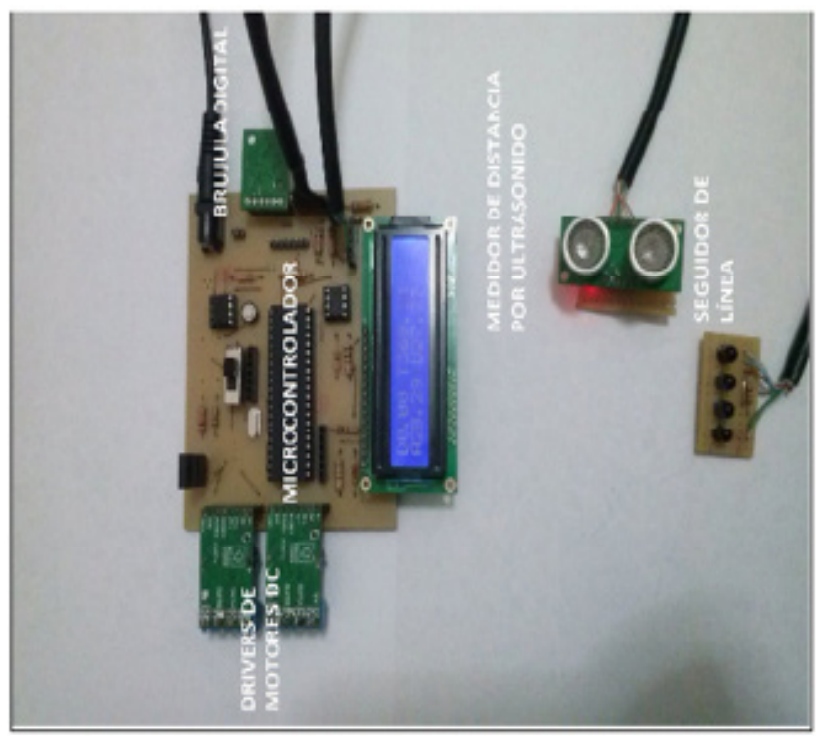

Fig. 8. Tarjeta electrónica para la gestión de la sensórica del AGV

\section{Energía, drivers y otros}

Las tarjetas electrónicas y los sistemas electromecánicos del AGV se alimentan con baterías recargables. Se ha dispuesto de un arreglo de dos baterías de $12 \mathrm{~V}$ de plomo-ácido para alimentar los motores de tracción, del mecanismo cartesiano y del actuador lineal. Los motores utilizados son del tipo de corriente continua con caja reductora, servomotores y motores paso a paso, su control se realiza mediante drivers acoplados a las tarjetas electrónicas del AGV. Para efectos de respaldo y de aislamiento del ruido debido a las maniobras de encendido de los motores se utilizan baterías recargables de $8.4 \mathrm{~V}$ para alimentar las tarjetas electrónicas del AGV. También se han tomado previsiones de diseño para incluir elementos de protección contra condiciones de sobrecorriente, sobrevoltaje y polarización inversa.

\section{Interfaces de usuario}

A la fecha se han desarrollado interfaces para computador utilizando el software de instrumentación virtual Labview, Fig. 9, y se avanza en el desarrollo de interfaces de usuario para dispositivos móviles con sistema operativo Android.

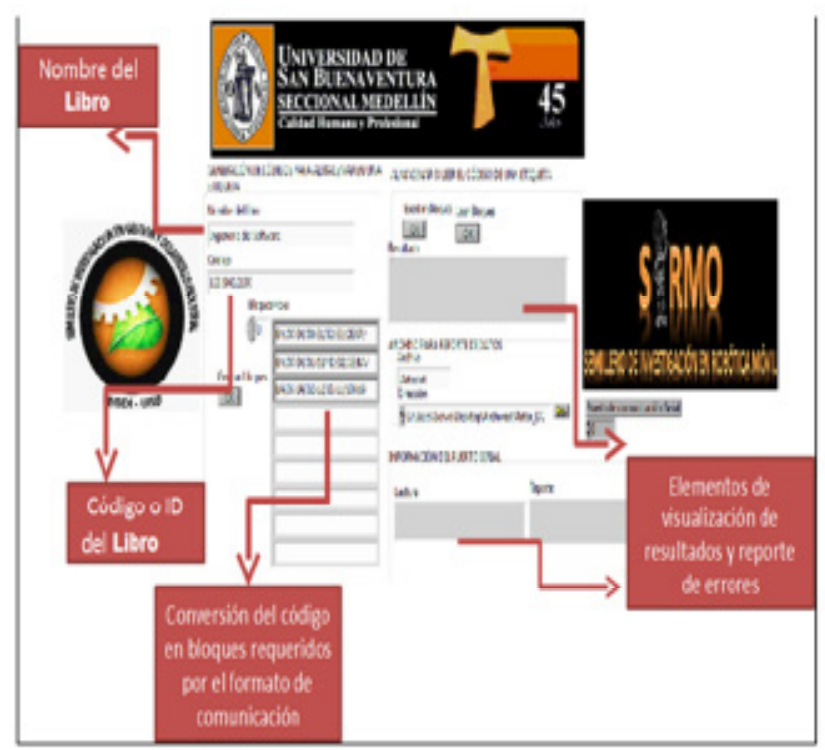

Fig. 9. Interfaz en Labview para lectura/escritura de etiquetas RFID para los libros del ASRS 


\section{CONCLUSIONES}

Se ha desarrollado un prototipo de vehículo de guiado autónomo que combina elementos mecánicos y electrónicos. Se procede ahora a la verificación de las capacidades de manipulación y transporte de inventario de esta estructura mecatrónica, para esto se avanza en la integración de los sistemas de procesamiento, sensórica, control y comunicaciones. El prototipo de vehículo de guiado autónomo deberá alcanzar la autonomía requerida para operar en el ambiente controlado, realizando adecuadamente las tareas propias del sistema de almacenamiento/recuperación de libros.

El protocolo MiWi P2P, que cubre las comunicaciones inalámbricas entre el AGV y los elementos circundantes del ASRS, ha demostrado adaptarse satisfactoriamente a las condiciones del ambiente de prueba del prototipo desarrollado. Una de las ventajas verificadas es su facilidad de implementación, debido a su footprint liviano en términos de memoria, lo que permite implementarlo en microcontroladores de 8 bits. El desarrollador puede modificar de manera ágil el firmware programado en los microcontroladores, desde la configuración de recursos pertenecientes a la capa controlador de acceso al medio (MAC) hasta el nivel de aplicación.

Actualmente se trabaja en la implementación de un sistema que permita la referenciación coordenada de la posición del AGV en el ambiente de prueba. Dado que los módulos GPS no operan apropiadamente en instalaciones interiores, se pretende apoyarse en otras alternativas, lo que imponen un reto tecnológico adicional para el sistema propuesto. Las cualidades del protocolo MiWi P2P se aprovecharán para desplegar nodos adicionales en el entorno del ASRS para apoyar las tareas de referenciación coordenada del AGV. En las fases siguientes del proyecto se enfatizará en el desarrollo de interfaces de usuario para dispositivos móviles con sistema operativo Android, esto se visualiza como una oportunidad para añadir valor agregado al ASRS propuesto.

\section{BIBLIOGRAFÍA}

1. S. Hossain, M. Y. Ali, H. Jamil, and M. Z. Haq, "Automated guided vehicles for industrial logistics - Development of intelligent prototypes using appropriate technology," in The 2nd International Conference on Computer and Automation Engineering (ICCAE), 2010, vol. 5, pp. 237-241.

2. Rashid, M.M.; Kasemi, B.; Rahman, M., "New Automated Storage and Retrieval System (ASRS) using wireless communications," Mechatronics (ICOM), 2011 4th International Conference On , vol., no., pp.1,7, 17-19 May 2011

3. S. Berman, E. Schechtman, and Y. Edan, "Evaluation of automatic guided vehicle systems," Robotics and Computer-Integrated Manufacturing, vol. 25, no. 3, pp. 522-528, Jun. 2009.

4. TAG Italia, "SL 015B-3 modulo lettore/scrittore," 2011.

5. Microchip Technology inc., "Microchip MiWi P2P Wireless Protocol," 2010.

6. Rovin Networks, "RN-41 / RN-41-N Class 1 Bluetooth Module," 2012 